\title{
Chitosan Derivatives/Calcium Carbonate Composite Capsules Prepared by the Layer-by-Layer Deposition Method II Stabilization of the Shell by Crosslinking
}

\author{
Takashi Sasaki, Hiroto Mizuuchi, and Kensuke Sakurai \\ Department of Materials Science and Engineering, University of Fukui, Fukui 910 8507, Japan \\ Correspondence should be addressed to Takashi Sasaki, sasaki@matse.u-fukui.ac.jp \\ Received 27 March 2011; Accepted 9 June 2011 \\ Academic Editor: Xiaogong Wang
}

Copyright ( 2011 Takashi Sasaki et al. This is an open access article distributed under the Creative Commons Attribution License, which permits unrestricted use, distribution, and reproduction in any medium, provided the original work is properly cited.

\begin{abstract}
The layer-by-layer deposition method is utilized to prepare rodlike core/shell capsules. Chitosan (polycation) and chitosansulfate (polyanion) were alternatively deposited on the surface of calcium carbonate whisker (rodlike particle). The thickness of the obtained shells ranged from 26 to $42 \mathrm{~nm}$. After the deposition, the shell was treated with diisocyanate to form crosslink between the chitosan or chitosansulfate chains in order to stabilize the deposited shell. The obtained shell crosslinked rodlike capsules were successfully converted to hollow particles by immersing them into hydrochloric acid due to the enhanced shell stability, whereas from noncrosslinked shells, no hollow capsules were yielded: the shell was removed by dissolution in hydrochloric acid. It is revealed that the crosslinking reactivity is higher for 1,6-diisocyanatehexane than for tolylene 2,4-diisocyanate, suggesting that the reactivity depends on the size and flexibility of the crosslinking molecule.
\end{abstract}

\section{Introduction}

The layer-by-layer deposition method is one of the most versatile and promising techniques to fabricate encapsulated nanoparticles [1], which can be used as diverse applications such as drug delivery system. In this technique, oppositely charged polyelectrolytes are alternatively deposited on a substrate or surface of template particle to construct a thin multilayered film or shell [2-6]. In general, the efficiency of the deposition depends on the chemistry and molecular weight of the polyelectrolytes, the ion strength, the $\mathrm{pH}$ value, and temperature [7-11]. Optimization of these deposition conditions is required to fabricate multilayers that have prescribed structures for various purposes.

We have prepared various rodlike capsules consisting of a calcium carbonate core (whisker) and a polymer thin shell with the thickness of thinner than $100 \mathrm{~nm}$ by the suspension polymerization method. The thermal properties and dynamics of the yielded nanosized thin shells have also been studied [12-14]. Furthermore, we have developed the layer-by-layer technique to make rodlike capsules that have chitosan/chitosansulfate multilayered shells [15]. Chitosan is a derivative of chitin, a natural polysaccharide; thus the capsules with the chitosan/chitosansulfate shell are expected to be used as biocompatible and biodegradable nanomaterials. The chitosan/chitosansulfate/calcium carbonate capsules can be converted to hollow particles by removing the core via a simple acid treatment: calcium carbonate can be readily dissolved in hydrochloric acid. The hollow capsules thus yielded may be further converted to capsules possessing various inorganic cores such as ferrites, leading to broader applications of the capsules [16]. However, the deposited shells are unstable especially for a relatively low molecular weight chitosan of less than $10^{5}$. Although complexation occurs in the multilayered shell prepared by the layer-bylayer method, lower molecular weight shells are actually dissolved in hydrochloric acid, which prevents the formation of hollow capsules. We have tried to crosslink the chitosan shell by photodimerization of cinnamoyl groups which were introduced in the chitosan chain, but sufficient stability of the shell was not achieved [15].

In this study, we further investigate the stabilization of the shell via crosslink. Here we use two diisocyanates, 1,6diisocyanatohexane (HDMI), and tolylene 2,4-diisocyanate 
(TDI), as crosslinking reagents. A low molecular weight chitosan $\left(\mathrm{ca} .10^{5}\right.$ ) is used to check the stabilization effect of resulted crosslink. Isocyanate group can react with either amino or hydroxyl groups of chitosan. Expected crosslinking reaction is presented in Scheme 1.

\section{Experimental Methods}

2.1. Materials. Chitosan $\left(M_{w}=9.7 \times 10^{4}\right)$ was supplied from Dainichiseika Color \& Chemicals Manufacturing Co., Japan, was purified by reprecipitation from a $1.0 \%$ acetic acid aqueous solution in a $10 \%$ aqueous solution of sodium hydroxide, and was washed with distilled water, methanol, acetone, and N,N-dimethyl formamide (DMF). Calcium carbonate whisker was supplied from Maruo Calcium Co., Japan, of which the diameter ranged from 300 to $600 \mathrm{~nm}$, and the length ranged from 1.0 to $3.0 \mu \mathrm{m}$. The surface of the whisker was covered with a thin layer of calcium phosphate by phosphoric acid treatment that was performed by the manufacturer. Chitosansulfate was synthesized from chitosan by a reaction with chlorosulfonic acid in a DMF solution. Detailed procedure of the synthesis is described in the previous paper [15]. The number of sulfonic groups per repeating unit of chitosan was 0.86 . HDMI and TDI were purchased from Aldrich and were used without further purification.

2.2. Layer Deposition. We first prepared aqueous solutions of chitosan and chitosansulfate. Chitosan (25 mg) was dissolved in distilled water $(50 \mathrm{~mL})$ with sodium chloride $\left(1.46 \mathrm{~g}, 0.50 \mathrm{~mol} \mathrm{~L}^{-1}\right)$ and acetic acid $(0.50 \mathrm{~mL}, 1.0 \% \mathrm{v} / \mathrm{v})$. The chitosan content of this solution (referred to as A) was revealed to be $0.50 \mathrm{~g} \mathrm{~L}^{-1}$. Chitosansulfate $(26.5 \mathrm{mg}$ ) was added to distilled water $(50 \mathrm{~mL})$ with sodium chloride $\left(1.46 \mathrm{~g}, 0.50 \mathrm{~mol} \mathrm{~L}^{-1}\right)$. After stirring for $1 \mathrm{~h}$ at room temperature, insoluble fraction due to gelation was removed by filtration. The yielded homogeneous solution is referred to as $\mathrm{B}$, of which the polymer content was revealed to be $0.24 \mathrm{~g} \mathrm{~L}^{-1}$.

Layer-by-layer deposition of chitosan (cationic polyelectrolyte) and chitosansulfate (anionic polyelectrolyte) was executed by the membrane filtration method [17]; the suspended state of particles was maintained throughout the deposition-filtration-lavage-filtration cycle to avoid particle aggregation; this included no separation process of the particles from the suspension. Calcium carbonate whisker $(2.0 \mathrm{~g})$ was well suspended in distilled water $(60 \mathrm{~mL})$ with sonication, and then, the solution $\mathrm{A}(7.5 \mathrm{~mL})$ was added. The suspension was stirred for $5 \mathrm{~min}$ for deposition of a chitosan layer on the whisker surface. The suspended particles were then washed with distilled water under constant stirring to keep the well-suspended state. This was performed on a glass filter, where the liquid phase (chitosan solution) was exchanged for distilled water by controlling the rates of water addition and draining. Subsequently, the solution B $(17 \mathrm{~mL})$ was added to the suspension, which was stirred for $5 \mathrm{~min}$ for the deposition of chitosansulfate layer. The suspension was again washed with distilled water as above. The above cycle of deposition with the solutions A and B was repeated 0.5-12 times ( 0.5 means that just single chitosan layer is deposited).
The total number of deposited layer $m$ ranged from 1 to 24 . Additionally, we also prepared a capsule possessing only a single layer of chitosansulfate $(m=1)$. Finally, the capsules were separated by membrane filtration $(0.2 \mu \mathrm{m})$ and were dried in vacuum for $48 \mathrm{~h}$ at room temperature. No significant loss of the particles through the filtration was observed.

2.3. Crosslinking. Shell crosslinked capsules were prepared from those of which the outermost layer is chitosan, that is, the capsules with odd number of $m$. The core/shell capsule $(1.0 \mathrm{~g})$ was redispersed in cyclohexane $(100 \mathrm{~mL})$ with sonication. HMDI $(0.17 \mathrm{~g})$ or TDI $(0.13 \mathrm{~g})$ was added to this suspension, and it was stirred for $3 \mathrm{~h}$ at room temperature. The reacted particles were then separated by membrane filtration $(0.2 \mu \mathrm{m})$ and were washed with cyclohexane for 10 times to remove unreacted diisocyanate.

2.4. Preparation of Hollow Capsules. The prepared core/shell capsule $(1.0 \mathrm{~g})$ was added to $3.4 \%$ hydrochloric acid $(50 \mathrm{~mL})$ and was stirred for $2 \mathrm{~h}$ confirming that the $\mathrm{pH}$ was below 1.0, until the core part (calcium carbonate) was completely dissolved. The particles were separated by centrifugation and were washed with water several times. The yielded particles were dried under vacuum for more than $48 \mathrm{~h}$ at room temperature.

2.5. Characterization. Morphology of the capsules was examined by transmission electron microscopy (TEM) with a Jeol JEM-2000FXII operated with an acceleration voltage of $160 \mathrm{kV}$. The average shell thickness and its standard deviation were evaluated from the electron micrographs by measuring ca. 100 different locations of different particles. Fourier transform infrared spectroscopy was performed by using a Nicolet Magna 560 spectrometer.

\section{Results and Discussion}

Figure 1 shows electron micrographs for the core/shell (filled) and hollow capsules with a crosslinked chitosan shell of $m=1$. The hollow shells are well formed for crosslinked samples, whereas no hollow shell was yielded from a noncrosslinked capsule with $m=1$ as has been expected: chitosan is soluble in hydrochloric acid. On the contrary, the crosslinked shells are not dissolved in the acid solution though some swelling may have occurred, resulting in the hollow capsules as shown in Figures 1(b) and 1(d). We occasionally found partial lack of core material in the tip part of the rodlike particle as shown in Figure 1(c). The mechanism of this is not clear, but we infer that it occurred during the purification (stirring) process after the shell deposition.

In the multilayered shells prepared by the layer-by-layer deposition, complexation between the two polyelectrolytes with opposite charges is considered to occur, which can prevent dissolution in the acidic solution during the core removing process. Indeed, we have reported that core/shell capsules with a high molecular weight chitosan/chitosansulfate $\left(1.09 \times 10^{6}\right)$ shells give hollow capsules even without crosslinking [15]. On the contrary, capsules with a lower molecular weight chitosan/chitosansulfate $\left(9.7 \times 10^{4}\right)$ used 


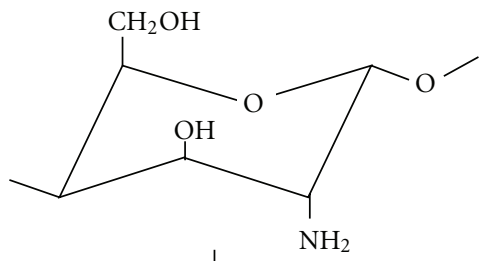

HMDI: $-\mathrm{R}-=-\left(\mathrm{CH}_{2}\right)_{6}-$

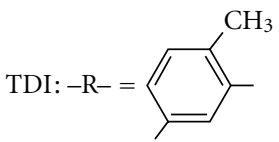

OCN-R-NCO

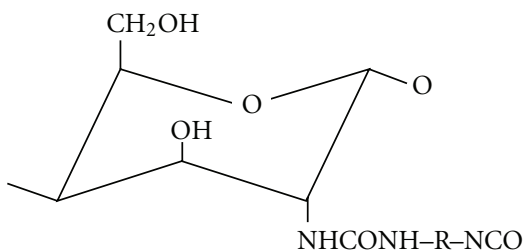

Or
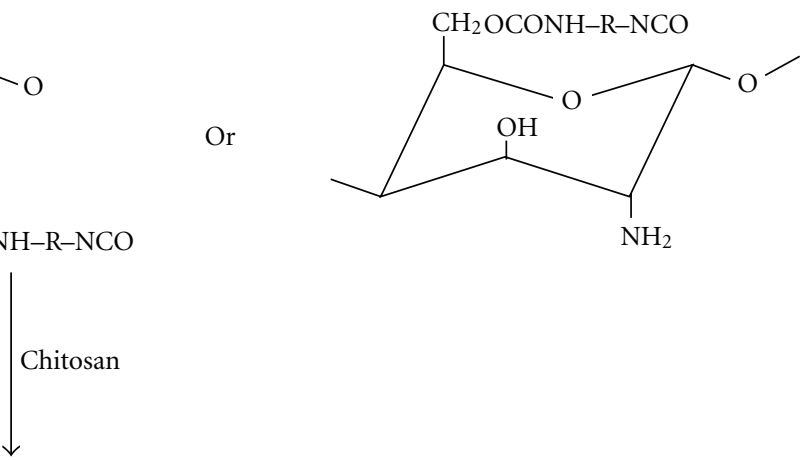

Crosslinked chitosan

Scheme 1: Crosslinking by diisocyanate.

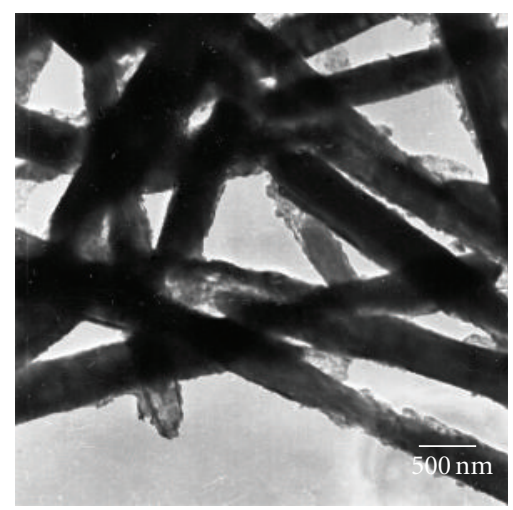

(a)

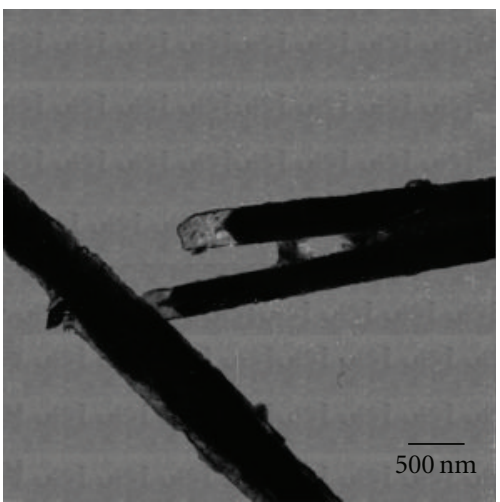

(c)

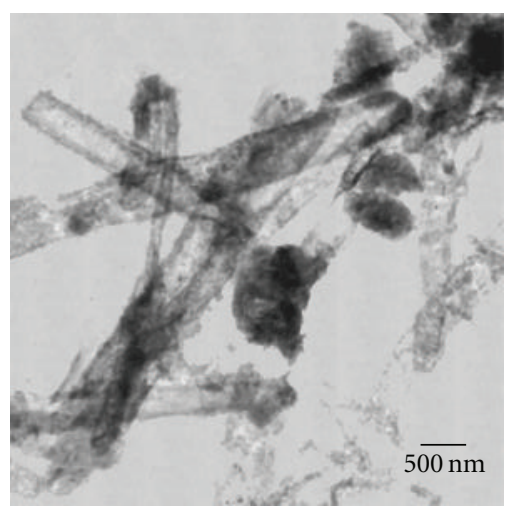

(b)

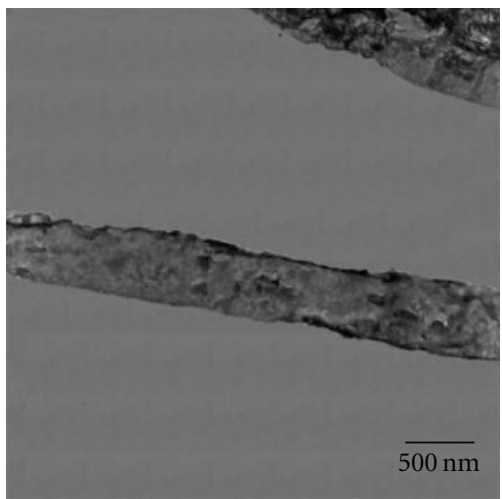

(d)

FIGURE 1: Transmission electron micrographs for capsules with a crosslinked chitosan shell $(m=1)$. (a) A filled capsule crosslinked by HMDI, (b) a hollow capsule crosslinked by HMDI, (c) a filled capsule crosslinked by TDI, and (d) a hollow capsule crosslinked by TDI. 


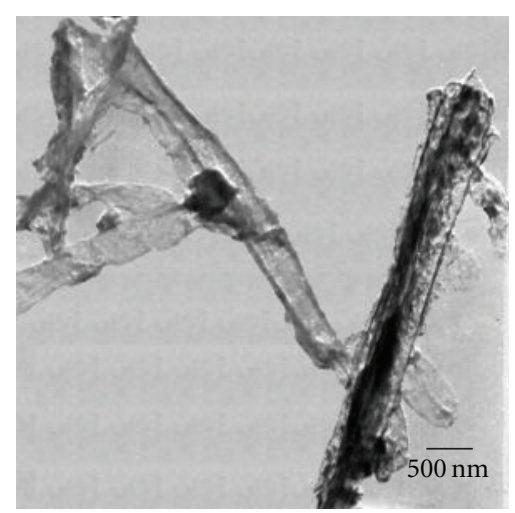

(a)

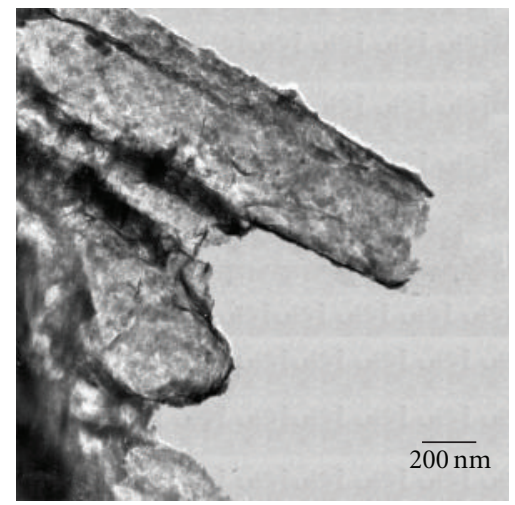

(c)

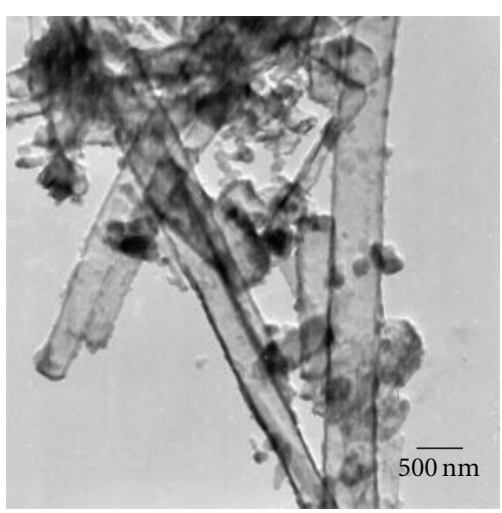

(b)

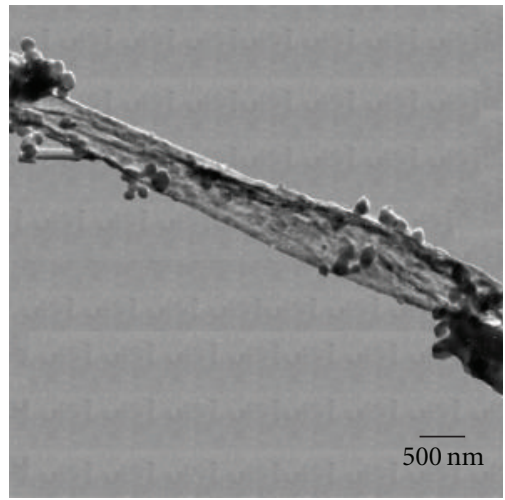

(d)

FIGURE 2: Transmission electron micrographs for hollow capsules with a crosslinked chitosan/chitosansulfate shell prepared by the layer-bylayer deposition method. (a) A capsule of $m=5$ crosslinked by HMDI, (b) $m=5$ crosslinked by TDI, (c) $m=11$ crosslinked by HMDI, and (d) $m=11$ crosslinked by TDI.

in the present study did not give hollow capsules without crosslinking. This result may be explained as follows. The complexation during the deposition does not fully occur due to the nonuniformity of the deposition which is discussed later, and the fraction of polymer without complexation tends to be redissolved in the acidic solution. This tendency is prominent for lower molecular weight polymers because of their higher solubility and lower connectivity.

Figure 2 shows hollow capsules with crosslinked shells of chitosan/chitosansulfate. Hollow structure is well formed for both the two crosslinking reagents, HMDI and TDI. This indicates that the crosslink prevents the dissolution even if the complexation may not be perfect. We thus confirmed that the crosslink is effective in stabilizing the core/shell and hollow structures of the capsule.

We found that the thickness $l$ of filled capsules without crosslink increases linearly with $m$, obeying a relation $l=$ $m d+a$, where $d=1.3 \mathrm{~nm} /$ layer and $a=26 \mathrm{~nm}$ for the present deposition system. The nonzero $a$ value means that the mechanism of the first layer (chitosan) is different from that for the subsequent depositions as discussed in the previous paper [15]. The value of $d$, which is the thickness per one layer for $m>1$, is much greater than that expected for a monolayer of chitosan (a few angstroms), suggesting that the deposition is associated with some aggregation of the polymer material. As we have reported previously, the obtained hollow capsules are rather rigid, retaining their original rodlike shape [15]. This is probably due to the rigidity of the chitosan chain $[18,19]$. We also found that for the present deposition system, the thickness did not increase any more when $m$ exceeded 22. As $m$ increases, nonuniformity of the deposition due aggregation or incompleteness of the complexation becomes prominent, and the layer deposition with uniform complexation may become difficult to occur.

Table 1 shows the thickness of the shell which was estimated from the TEM micrographs. The $l$ values of crosslinked and hollow shells approximately obey the above linear relation, indicating that the crosslinking and core removing treatments do not affect the thickness very much. This suggests that the crosslink formation occurs not only in the outermost layer but also in the interior layers; otherwise the thickness would be reduced upon removing the core by dissolution. It should be remembered that from the low molecular weight shells, no hollow capsules are yielded because of higher solubility in hydrochloric acid. Additionally, we consider that the crosslink reaction largely occurs within each particle: the possibility of inter-particle crosslinking may be subtle because the reaction was performed in a well-suspended state with constant stirring. 
TABLE 1: Thickness of the shell.

\begin{tabular}{lccccc}
\hline Shell $^{(1)}$ & $\begin{array}{c}\text { Crosslinking } \\
\text { reagent }\end{array}$ & Filled/hollow & $m$ & $l(\mathrm{~nm})$ & $\sigma_{l}(\mathrm{~nm})^{(2)}$ \\
\hline $\mathrm{C}$ & Non & Filled & 1 & 27 & 11 \\
$\mathrm{C}$ & HMDI & Filled & 1 & 28 & 6 \\
$\mathrm{C}$ & HMDI & Hollow & 1 & 26 & 9 \\
$\mathrm{C}$ & TDI & Filled & 1 & 21 & 10 \\
$\mathrm{C}$ & TDI & Hollow & 1 & 22 & 8 \\
$\mathrm{C} / \mathrm{S}$ & HMDI & Hollow & 5 & 35 & 9 \\
$\mathrm{C} / \mathrm{S}$ & TDI & Hollow & 5 & 38 & 9 \\
$\mathrm{C} / \mathrm{S}$ & HMDI & Hollow & 11 & 42 & 8 \\
$\mathrm{C} / \mathrm{S}$ & TDI & Hollow & 11 & 39 & 11 \\
$\mathrm{~S}$ & HMDI & Hollow & 1 & 26 & 10 \\
\hline
\end{tabular}

${ }^{(1)}$ C: chitosan, S: chitosansulfate.

${ }^{(2)} \sigma_{l}$ : standard deviation of $l$.

The diisocyanates employed in this study may react either with amino or hydroxyl group in chitosan as shown in Scheme 1. In the former reaction results in a urea group $(-\mathrm{NH}(\mathrm{C}=\mathrm{O}) \mathrm{NH}-)$, while the latter leads to a urethane group $(-\mathrm{O}(\mathrm{C}=\mathrm{O}) \mathrm{NH}-)$. Figure 3 shows infrared spectra for the crosslinked hollow shells with $m=1$. The shell crosslinked with HMDI exhibits absorption bands at 1570 and $1630 \mathrm{~cm}^{-1}$, which are assigned to a urea group (amide and carbonyl groups) [20]. Similarly, the shell crosslinked with TDI exhibits absorption bands at 1597 and $1650 \mathrm{~cm}^{-1}$ that are also assigned to a urea group. On the contrary, no absorption signals due to urethane group are observed at 1698 and $1709 \mathrm{~cm}^{-1}$, the former being characteristic of hydrogen bonding between a urethane carbonyl and a urea amide, and the latter of a hydrogen-bonded urethane carbonyl. This may be due to the higher reactivity of the isocyanate with the amino group than with the hydroxyl group of chitosan.

Although excess $-\mathrm{NCO}$ group against $-\mathrm{NH}_{2}$ was fed in the reaction (the ratio $\mathrm{NCO} / \mathrm{NH}_{2}=1.4-5.9$ ), the reaction with hydroxyl group did not occur. This suggests that the reaction efficiency is relatively low due to low molecular mobility in the solid phase (shell), which may be inherent to such a liquid-solid heterogeneous reaction.

We also found that the crosslinking reactivity is higher for HMDI than for TDI. Table 2 shows relative absorbance of the infrared bands characteristic of the urea group. Here the absorbance for each band is divided by that at $1030 \mathrm{~cm}^{-1}$ which is characteristic of original chitosan. The result indicates that the formation of urea is greater by a factor of ca. 1.2 for the shell crosslinked by HMDI than by TDI. We also found that the shell crosslinked by TDI exhibits a band at $2280 \mathrm{~cm}^{-1}$, which is due to unreacted isocyanate group, whereas for the shell crosslinked by HMDI, the band was not observed. In HMDI, the two isocyanate groups are separated by a flexible methylene chain, whereas in TDI, they are fixed to a small rigid phenyl group and have less degree of freedom regarding the distance and relative orientation between the two isocyanategroups, which seems to be

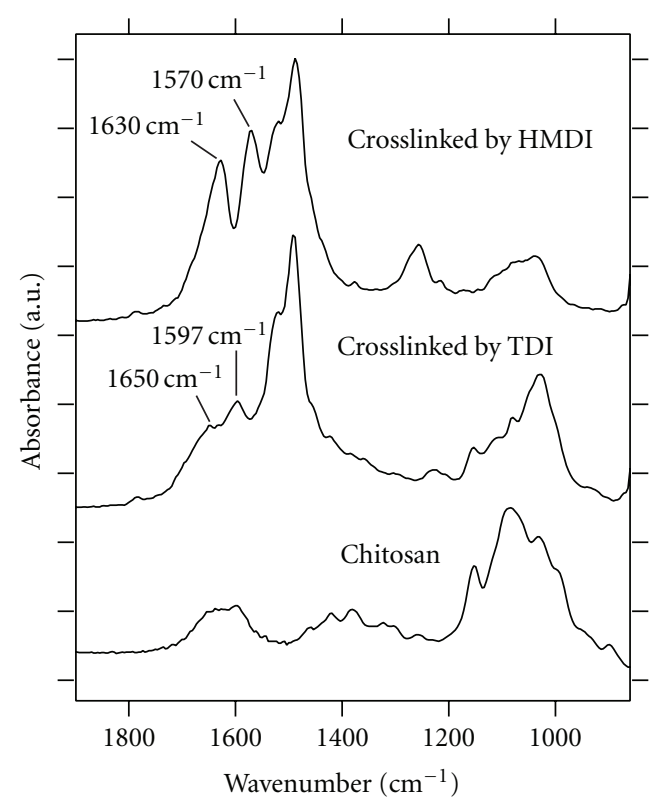

FIGURE 3: Infrared spectra for the hollow chitosan capsules crosslinked by HMDI and TDI, and for original chitosan (as a reference). The positions on the vertical axis are shifted to enable the profiles to be distinguished.

TABLE 2: Relative infrared absorbance $A(\nu) / A\left(1030 \mathrm{~cm}^{-1}\right)$ due to the urea group for crosslinked chitosan shell $(m=1)$.

\begin{tabular}{lcc}
\hline $\begin{array}{l}\text { Crosslink } \\
\text { reagent }\end{array}$ & $v=1572$ or $1597 \mathrm{~cm}^{-1}$ & $v=1630$ or $1650 \mathrm{~cm}^{-1}$ \\
\hline HMDI & 1.18 & 1.14 \\
TDI & 0.96 & 0.92 \\
\hline
\end{tabular}

disadvantageous for the present crosslinking. Nevertheless, the fact that crosslinking with TDI still yields hollow capsule suggests that the reactive groups are rather concentrated in the deposited shell. We also tried tolylene 2,5-diisocyanate as another crosslinking reagent, but no apparent sign of reaction was observed from the infrared spectroscopy, and no hollow capsules were yielded. This suggests that the crosslinking reaction is sensitive to the relative location and orientation of the two isocyanate groups.

Figure 4 shows micrographs of the capsules with chitosansulfate shell $(m=1)$ crosslinked by HMDI. Similar to chitosan and chitosan/chitosansulfate shells, hollow capsules are well formed, suggesting that the crosslinking is successfully occurred even in the chitosansulfate shell. Considering that the present chitosansulfate still contains free amino and hydroxyl groups (the number of sulfonic groups per one glucosamine unit is 0.86 ), the crosslink reaction can take place. Here again, it is demonstrated that the crosslink stabilizes the shell structure. The thickness of the hollow shell is $26 \mathrm{~nm}$, which is similar to that for the chitosan shell with $m=1$ (see Table 1 ). 


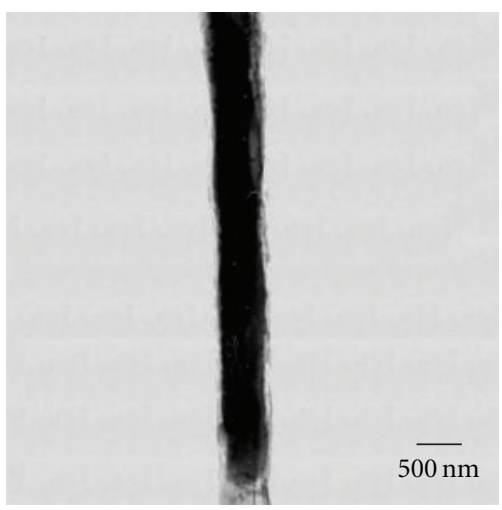

(a)

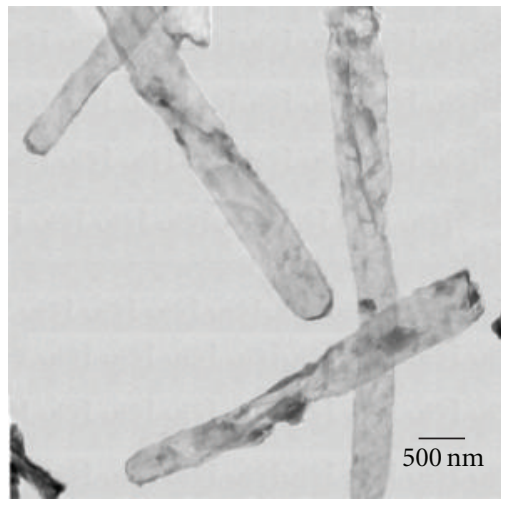

(b)

FIgURE 4: Transmission electron micrographs for the capsules with a chitosansulfate shell $(m=1)$ crosslinked by HMDI. (a) A filled capsule and (b) a hollow capsule.

\section{Conclusions}

We have shown that the chitosan, chitosan/chitosansulfate, and chitosansulfate thin shells can be crosslinked by diisocyanates HMDI and TDI, which stabilizes the shell structure. The stabilization effect is evidenced by the formation of hollow capsules even for $M_{w}=9.7 \times 10^{4}$ : hollow capsules cannot be obtained without crosslinking for this molecular weight. HMDI exhibits higher reactivity than TDI by a factor of ca. 1.2 as revealed by the relative absorbance of the infrared band due to the urea group. The present results show that crosslinking is an effective technique to fabricate stable core/shell particles. From the obtained hollow shells stabilized with crosslink, core/shell capsules possessing different core materials can be prepared via, for example, selective precipitation of ferrite inside a hollow particle where the $\mathrm{pH}$ is controlled. This is currently under investigation.

\section{Acknowledgments}

This work was supported by a Grant-in-Aid for Scientific Research (no. 18550107) from the Ministry of Education, Culture, Sports, Science and Technology of Japan. The authors thank Yuki Tsujimoto for help in sample preparation.

\section{References}

[1] G. Decher, "Layered nanoarchitectures via directed assembly of anionic and cationic molecules," in Comprehensive Supramolecular Chemistry, J.-P. Sauvage and M. W. Hosseini, Eds., vol. 9, Pergamon Press, Oxford, UK, 1996.

[2] I. L. Radtchenko, G. B. Sukhorukov, S. Leporatti, G. B. Khomutov, E. Donath, and H. Möhwald, "Assembly of alternated multivalent ion/polyelectrolyte layers on colloidal particles. Stability of the multilayers and encapsulation of macromolecules into polyelectrolyte capsules," Journal of Colloid and Interface Science, vol. 230, no. 2, pp. 272-280, 2000.

[3] H. P. Yap, J. F. Quinn, A. P. R. Johnston, and F. Caruso, "Compositional engineering of polyelectrolyte blend capsules," Macromolecules, vol. 40, no. 21, pp. 7581-7589, 2007.

[4] J. H. Wosnick, J. H. Liao, and T. M. Swager, "Layer-by-layer poly(phenylene ethynylene) films on silica microspheres for enhanced sensory amplification," Macromolecules, vol. 38, no. 22, pp. 9287-9290, 2005.

[5] K. Katagiri, A. Matsuda, and F. Caruso, "Effect of UVirradiation on polyelectrolyte multilayered films and hollow capsules prepared by layer-by-layer assembly," Macromolecules, vol. 39, no. 23, pp. 8067-8074, 2006.

[6] M. Nakamura, K. Katagiri, and K. Koumoto, "Preparation of hybrid hollow capsules formed with $\mathrm{Fe} 3 \mathrm{O} 4$ and polyelectrolytes via the layer-by-layer assembly and the aqueous solution process," Journal of Colloid and Interface Science, vol. 341, no. 1, pp. 64-68, 2010.

[7] Y. Lvov, G. Decher, and G. Sukhorukov, "Assembly of thin films by means of successive deposition alternate layers of DNA and poly(allylamine)," Macromolecules, vol. 26, no. 20, pp. 5396-5399, 1993.

[8] Z. Sui, D. Salloum, and J. B. Schlenoff, "Effect of molecular weight on the construction of polyelectrolyte multilayers: stripping versus sticking," Langmuir, vol. 19, no. 6, pp. 24912495, 2003.

[9] B. Schoeler, S. Sharpe, T. A. Hatton, and F. Caruso, "Polyelectrolyte multilayer films of different charge density copolymers with synergistic nonelectrostatic interactions prepared by the layer-by-layer technique," Langmuir, vol. 20, no. 7, pp. 27302738, 2004.

[10] S. T. Dubas and J. B. Schlenoff, "Factors controlling the growth of polyelectrolyte multilayers," Macromolecules, vol. 32, no. 24, pp. 8153-8160, 1999.

[11] S. E. Burke and C. J. Barrett, "pH-responsive properties of multilayered poly(L-lysine)/hyaluronic acid surfaces," Biomacromolecules, vol. 4, no. 6, pp. 1773-1783, 2003.

[12] T. Sasaki, S. Kawagoe, H. Mitsuya, S. Irie, and K. Sakurai, "Glass transition of crosslinked polystyrene shells formed on the surface of calcium carbonate whisker," Journal of Polymer Science Part B, vol. 44, no. 17, pp. 2475-2485, 2006.

[13] T. Sasaki, M. Misu, T. Shimada, and M. Teramoto, "Glass transition and its characteristic length for thin crosslinked polystyrene shells of rodlike capsules," Journal of Polymer Science Part B, vol. 46, no. 19, pp. 2116-2125, 2008.

[14] T. Sasaki, R. Kuroda, M. Teramoto et al., "Glass transition properties of PMMA thin shells deposited on rodlike calcium carbonate particles," Polymer Journal, vol. 43, no. 5, pp. 464470, 2011. 
[15] T. Sasaki, M. Shimizu, Y. Wu, and K. Sakurai, "Chitosan derivatives/calcium carbonate composite capsules prepared by the layer-by-layer deposition method," Journal of Nanomaterials, vol. 2008, no. 1, Article ID 185632, 8 pages, 2008.

[16] D. G. Shchukin, I. L. Radtchenko, and G. B. Sukhorukov, "Synthesis of nanosized magnetic ferrite particles inside hollow polyelectrolyte capsules," Journal of Physical Chemistry B, vol. 107, no. 1, pp. 86-90, 2003.

[17] A. Voigt, H. Lichtenfeld, G. B. Sukhorukov et al., "Membrane filtration for microencapsulation and microcapsules fabrication by layer-by-layer polyelectrolyte adsorption," Industrial and Engineering Chemistry Research, vol. 38, no. 10, pp. 40374043, 1999.

[18] Y. Wu, T. Seo, S. Maeda, T. Sasaki, S. Irie, and K. Sakurai, "Circular dichroism induced by the helical conformations of acylated chitosan derivatives bearing cinnamate chromophores," Journal of Polymer Science Part B, vol. 43, no. 11, pp. 1354-1364, 2005.

[19] Y. Wu, T. Seo, T. Sasaki, S. Irie, and K. Sakurai, "Layered structures of hydrophobically modified chitosan derivatives," Carbohydrate Polymers, vol. 63, no. 4, pp. 493-499, 2006.

[20] N. Luo, D. N. Wang, and S. K. Ying, "Hydrogen bonding between urethane and urea: band assignment for the carbonyl region of FT i.r. spectrum," Polymer, vol. 37, no. 14, pp. 3045 3047, 1996. 

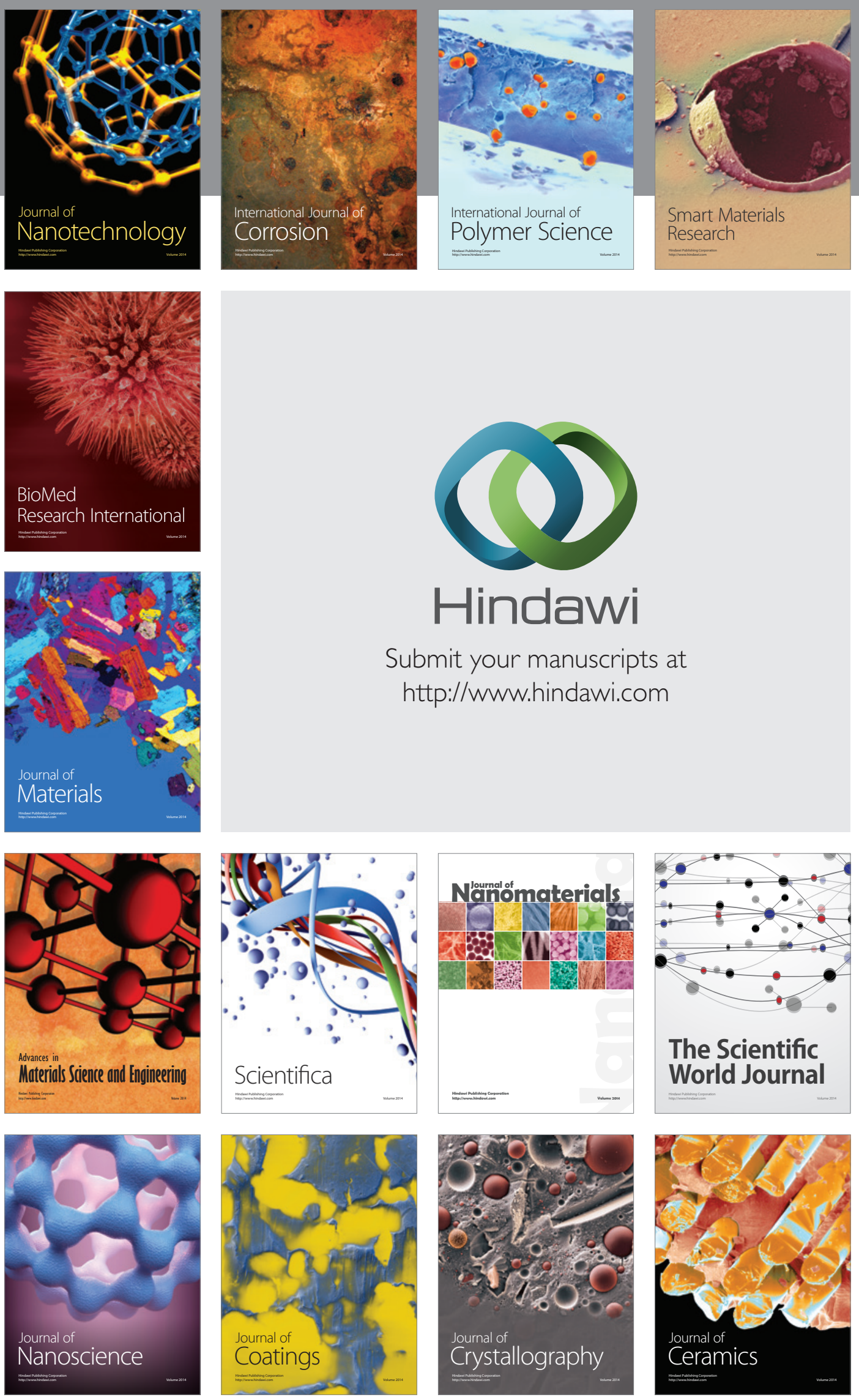

The Scientific World Journal

Submit your manuscripts at

http://www.hindawi.com

\section{World Journal}

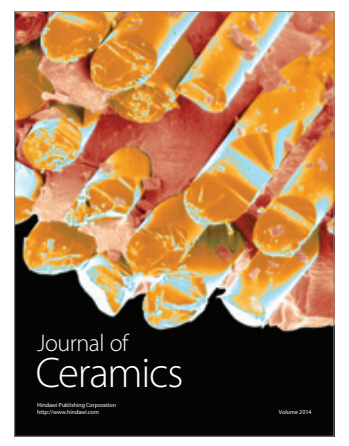

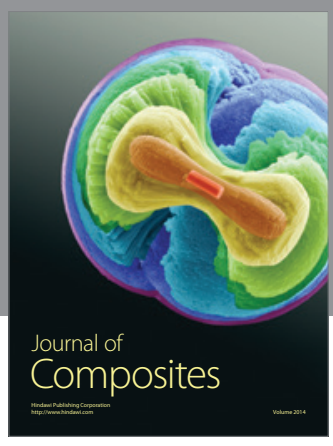
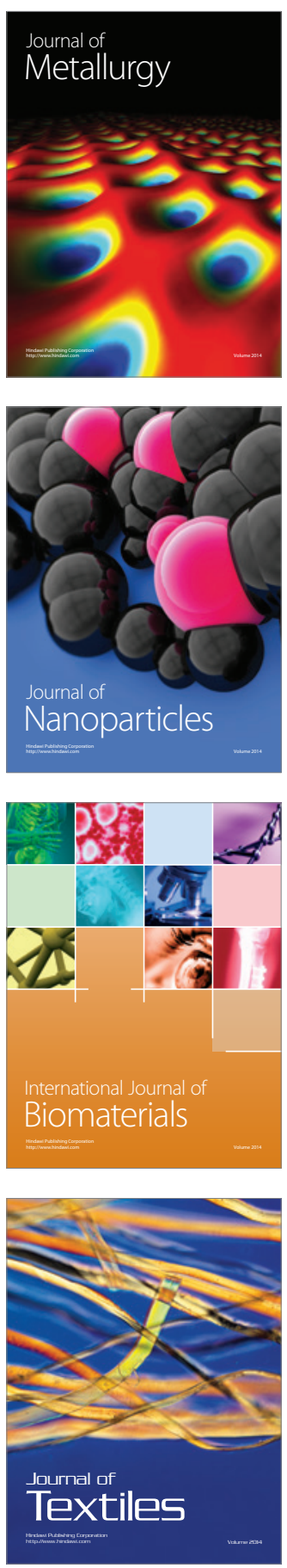